\title{
EFICÁCIA DA ACUPUNTURA E MOXABUSTÃO NO TRATAMENTO DE CADELA COM DOENÇA DO DISCO INTERVERTEBRAL: RELATO DE CASO
}

\author{
Ana Cristina dos Santos ${ }^{1}$ \\ Gelson Alcides dos Santos ${ }^{2}$ \\ Bruno Degaspari Minardi ${ }^{3}$ \\ Joanésia Maria Junkes Rothstein ${ }^{4}$
}

SANTOS, A. C. dos; SANTOS, G. A. dos; MINARDI, B. D.; ROTHSTEIN, J. M. J. Eficácia da acupuntura e moxabustão no tratamento de cadela com doença do disco intervertebral: relato de caso. Arq. Ciênc. Vet. Zool. UNIPAR, Umuarama, v. 18, n. 4, p. 247-251, out./dez. 2015.

\begin{abstract}
RESUMO: Existem relatos do uso terapêutico de acupuntura e moxabustão para a doença do disco intervertebral, muitas vezes, inclusive como terapia complementar. Neste estudo, relatou-se a eficácia da acupuntura e moxabustão no tratamento de uma cadela diagnosticada com doença do disco intervertebral. De acordo com a Medicina Tradicional Chinesa (MTC), o animal foi diagnosticado com deficiência de Yang do Rim, gerando deficiência de Qi do Baço e um quadro geral de Frio por deficiência, com acúmulo de Umidade. Para o tratamento, foram prescritas seis sessões semanais de acupuntura, além de estímulo dos acupontos com bastão de moxa três vezes por semana. A evolução do quadro foi evidente após a segunda sessão de acupuntura, com a melhora progressiva da propriocepção e da coordenação motora na marcha observadas antes mesmo da quinta sessão de acupuntura, apenas permanecendo uma leve ataxia. Assim, comprovou-se que a associação da acupuntura e moxabustão como tratamento de eleição de discopatia toracolombar em cão foi efetiva no restabelecimento da saúde do paciente. Considerando a visão holística da MTC, os benefícios da acupuntura e moxabustão incluem não só a solução da doença manifestada, como também o re-equilíbrio do indivíduo como um todo, ajudando no restabelecimento completo da saúde e bem-estar do paciente.
\end{abstract}

PALAVRAS-CHAVE: Discopatia. Beagle. Acuterapia. Moxaterapia.

\section{ACUPUNCTURE AND MOXIBUSTION EFFECTIVENESS IN TREATMENT OF FEMALE DOG WITH INTERVERTEBRAL DISC DISEASE: CASE REPORT}

\begin{abstract}
There are reports of the therapeutic use of acupuncture and moxibustion for the intervertebral disc disease, often including as complementary therapy. In this study, we have reported the efficacy of acupuncture and moxibustion in the treatment of a dog diagnosed with intervertebral disc disease. As the history and clinical signs, the animal had the presumptive diagnosis of thoracolumbar discopathy. According to Traditional Chinese Medicine (TCM), the animal was diagnosed with Kidney Yang deficiency generating Spleen Qi deficiency and a general clinical condition for cold by deficiency, with accumulation of Damp. For treatment, six sessions of acupuncture were required weekly, and stimulation of the acupoints with moxa stick three times a week. The evolution of the clinical condition was evident after the second acupuncture session, progressive improvement of proprioception and coordination in gait was observed even before the fifth session of acupuncture, remaining only a mild ataxia. Thus, it was shown that the combination of acupuncture and moxibustion as a treatment of choice for thoracolumbar disc disease in dog was effective in restoring the health of the patient. Considering the holistic view of TCM, the benefits of acupuncture and moxibustion include not only the solution of the manifested disease, as well as the re-balancing of the individual as a whole, helping in the full restoration of health and the patient's well-being.
\end{abstract}

KEYWORDS: Acutherapy. Beagle. Discopathy. Moxatherapy.

\section{EFICACIA DE LA ACUPUNTURA Y MOXIBUSTIÓN EN TRATAMIENTO DE PERRA CON ENFERMIDAD DE DISCO INTERVERTEBRAL: RELATO DE CASO}

RESUMEN: Hay informes sobre el uso terapéutico de la acupuntura y moxibustión para la enfermedad de disco intervertebral, a menudo incluido como terapia complementaria. En este estudio, se informó la eficacia de la acupuntura y la moxibustión en el tratamiento de una perra diagnosticado con enfermedad de disco intervertebral. Según la Medicina Tradicional China (MTC), el animal fue diagnosticado con deficiencia de Yang del Riñón generando deficiencia de Qi del Bazo y un cuadro general de Frío por discapacidad, con acumulo de Humedad. Para el tratamiento, fueron prescritas seis sesiones semanales de acupuntura, además de estímulos de los puntos de acupuntura con bastón de moxa tres veces a la semana. La evolución del cuadro clínico fue evidente después de la segunda sección de acupuntura, con mejora progresiva de la propiocepción y de

DOI: https://doi.org/10.25110/arqvet.v18i4.2015.5752

${ }^{1}$ Médica Veterinária - Biotério Central/Universidade Federal de Santa Catarina, Campus Reitor João David Ferreira Lima, Setor F, Santa Catarina. Email: ana.cristina@ufsc.br. Campus Reitor João David Ferreira Lima, Trindade, CEP88040-900, Florianópolis/SC.

${ }^{2}$ Técnico de Laboratório - Biotério Central/Universidade Federal de Santa Catarina

${ }^{3}$ Técnico de Laboratório - Biotério Central/Universidade Federal de Santa Catarina

${ }^{4}$ Coordenadora do Biotério Central/Universidade Federal de Santa Catarina 
la coordinación motora en marcha, observadas antes de la quinta sesión de acupuntura, permaneciendo solamente una ligera ataxia. Así, se comprobó que la combinación de la acupuntura y moxibustión como tratamiento elegido de la enfermedad discal toracolumbar en perro ha sido eficaz en la restauración de la salud del paciente. Considerando la visión holística de la MTC, los beneficios de la acupuntura y moxibustión incluyen no solamente la solución de la enfermedad manifestada, así como el reequilibrio del individuo en su conjunto, lo que ayuda el restablecimiento de la salud y bienestar del paciente.

PALABRAS CLAVE: Discopatía. Beagle. Acuterapia. Moxaterapia.

\section{Introdução}

A acupuntura e a moxabustão são alguns dos diversos métodos terapêuticos da Medicina Tradicional Chinesa (MTC). Como parte da MTC, ambas tem sua base fundamentada nos conceitos de Yin e Yang, dos cinco elementos, dos oito princípios, dos doze meridianos e das substâncias vitais (SCHWARTZ, 2008). Consideram o indivíduo e a doença como partes de um todo e atuam auxiliando o corpo na retomada do equilíbrio, com a manutenção dessa harmonia garantida pelo livre fluxo de Qi (LIMEHOUSE; TAYLOR-LIMEHOUSE, 2006). As indicações da acupuntura na Medicina Veterinária são amplas, incluindo distúrbios musculoesqueléticos, neurológicos, gastrointestinais, dermatológicos (SCHOEN, 2006).

Uma das doenças neurológicas mais comuns na prática clínica, a discopatia intervertebral é resultado da compressão do cordão espinhal, secundária a protusão/extrusão do disco intervertebral. A principal causa é a predisposição genética (raças condrodistróficas) e etária (três a sete anos) (BRAUND, 2010). Dependendo do grau das lesões presentes, os sintomas podem incluir ataxia, alteração postural devido à dor, paresia/paralisia dos membros e perda da percepção de dor. É diagnosticada por meio do histórico, sintomas e exames radiográficos, com o tratamento podendo ser conservativo e/ou cirúrgico (BRAUND, 2010; MASIAN, 2011).

Existem relatos do uso terapêutico de acupuntura e moxabustão para este distúrbio, sobretudo em humanos, porém, na Medicina Veterinária grande parte dos estudos é direcionada ao uso de acupuntura e eletroacupuntura, muitas vezes, como terapia complementar. Neste estudo, objetivou-se relatar a eficácia da acupuntura e moxabustão como tratamento de eleição em uma cadela diagnosticada com doença do disco intervertebral.

\section{Relato de caso}

Relata-se o caso clínico de uma cadela da raça $\mathrm{Be}$ agle, seis anos de idade, mantida no plantel reprodutivo do canil do Biotério Central da Universidade Federal de Santa Catarina. $\mathrm{O}$ animal convivia num box com mais duas fêmeas, com água ad libitum e era alimentado com ração seca, uma vez ao dia. Em 05/08/2014, a paciente apresentou sinais clínicos como vocalização (gemidos), andar atáxico e cifose na região toracolombar. Ao exame clínico, não foi observada exacerbação de dor ao toque nessa região. Aplicou-se moxabustão nos acupontos B23 e E36, com aparente redução na postura de cifose e na ataxia. Outras três aplicações de moxabustão foram efetuadas a cada dois dias nos pontos B23 e R3, observando-se uma melhora no quadro clínico.

Os mesmos sinais clínicos retornaram dia 03/09/2014 (cifose, vocalização em gemido, ausência de exacerbação de dor a palpação). No ambiente de alojamento foi observado presença de fezes pastosas. Devido a suspeita de dor abdominal decorrente de um distúrbio gastrointestinal inespecífico, realizou-se agulhamento seco em yintang, IG11, E25 e VC6, não sendo observados os referidos sintomas nos dias subsequentes.

No dia 26/09/2014, a cadela apresentou paralisia dos membros pélvicos, ausência de sensibilidade à dor profunda e de exacerbação de dor a palpação da região toracolombar (Figura 1). Havia fezes diarreicas no box onde o animal estava alojado. Ao exame clínico, ainda apresentou língua de coloração pálido-roxa e secreção marrom nos ouvidos.

Figura 1: Paciente com diagnóstico de discopatia, após primeira sessão de acupuntura e moxabustão.

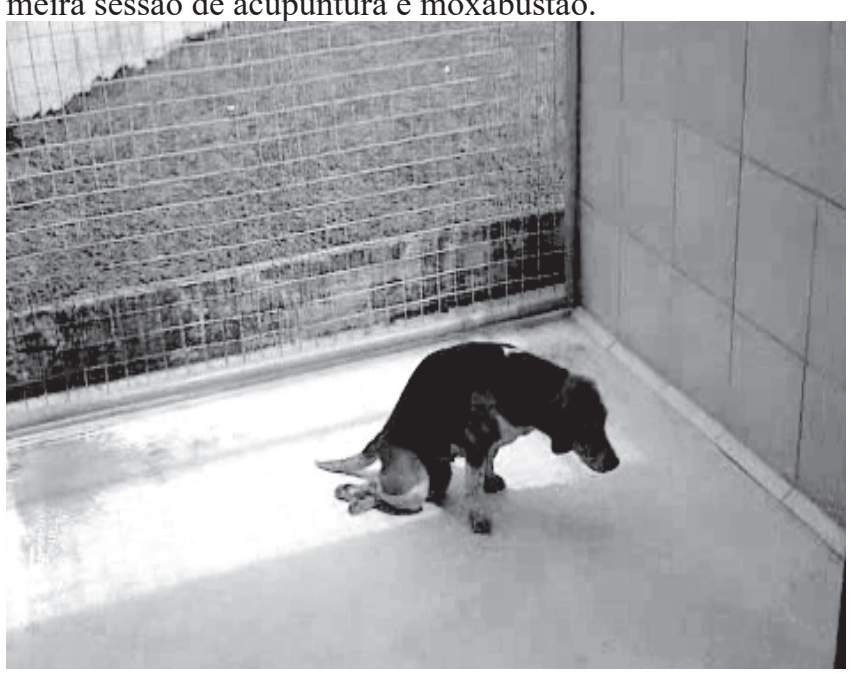

Fonte: Arquivo pessoal.

De acordo com o histórico (raça condrodistrófica e a idade) e sinais clínicos (postura em cifose e andar atáxico, com evolução para paralisia dos membros pélvicos), o animal teve o diagnóstico presuntivo de doença do disco intervertebral na região das vértebras toracolombares. Não foi realizado exame radiográfico. Com base na MTC, o diagnóstico do quadro foi deficiência de Yang do rim, gerando deficiência de Qi do baço e um quadro geral de Frio por deficiência, com acúmulo de umidade. O tratamento preconizado de acordo com a MTC foi tonificar o Yang do rim e o Qi do baço e eliminar a Umidade. Para isso, foram prescritas inicialmente seis sessões semanais de acupuntura (agulhamento seco) ${ }^{1}$, além de estímulo dos acupontos com bastão de moxa ${ }^{2}$ três vezes por semana em pontos dos meridianos da Bexiga (B), Rim (R), Estômago (E), Baço-Pâncreas (BP), Vesícula biliar (VB), Vaso Governador (VG) e Vaso Concepção (VC), conforme Tabela 1. O tempo de estimulação nos acupontos foi de dez minutos com as agulhas e três minutos com a moxa.

\footnotetext{
${ }^{1}$ Agulhas de aço inoxidável 0,25x30 e 0,25x15, Dong Bang, São Paulo - SP. ${ }^{2}$ Moxa bastão de Artemísia, Suzhou moxibustion supply factory, São Paulo $-\mathrm{SP}$
} 
Tabela 1: Acupontos utilizados durante o tratamento de cadela da raça Beagle, com seis anos de idade, diagnosticada com discopatia intervertebral, Florianópolis, 2014.

\begin{tabular}{c|l|l|l}
\hline Data & \multicolumn{1}{|c}{ Agulha } & Agulha+Moxa & \multicolumn{1}{c}{ Moxa } \\
\hline $26 / 09 / 14$ & yintang, R3, B60 & B23, E36 & VC4, VG4, B23, E36 \\
$28 / 09 / 14$ & & & VC4, VG4, B23, E36, B60 \\
$30 / 09 / 14$ & & VG4 \\
$03 / 10 / 14$ & yintang, VC4, VB39, B60, R3, B67 & B23 & VC4, VG4, B23, E36 \\
$06 / 10 / 14$ & & & VC4, VG4, B23, E36 \\
$08 / 10 / 14$ & & VG4 \\
$10 / 10 / 14$ & VC4, VB39, B60, R3, B67 & B23 & VC4, VG4, B23, E36 \\
$13 / 10 / 14$ & & & VC4, VG4, B23, E36, R3 \\
$15 / 10 / 14$ & yintang, BP9, VB39, B60, R3, B67 & B23 & VG4 \\
$17 / 10 / 14$ & & VC4, VG4, B23, E36, B60 \\
$20 / 10 / 14$ & & & VC4, VG4, B23, E36, B60 \\
$22 / 10 / 14$ & yintang, E39, R3, B67, BP9 & B23, B60 & VG4, E36 \\
$24 / 10 / 14$ & VC4, VG4, B23, E36, B60 \\
$29 / 10 / 14$ & & & VG4, E36 \\
$31 / 10 / 14$ & yintang, E40, R3, B60 & B23 & VG \\
\hline
\end{tabular}

Com a evolução do quadro clínico, as sessões foram reduzidas progressivamente para intervalos de quinze dias e posteriormente uma vez por mês. A melhora foi evidente após a segunda seção de acupuntura, com a cadela já conseguindo ficar em pé e dar alguns passos, apesar da dificuldade de se manter em estação (Figura 2). As fezes estavam normais, a secreção auricular reduzida e a melhora da propriocepção e da coordenação motora na marcha foram progressivas e observadas antes mesmo da quinta sessão de acupuntura, observando-se apenas a presença de uma leve ataxia.

Figura 2: Após duas semanas do início do tratamento, a paciente já retoma a capacidade de locomoção.

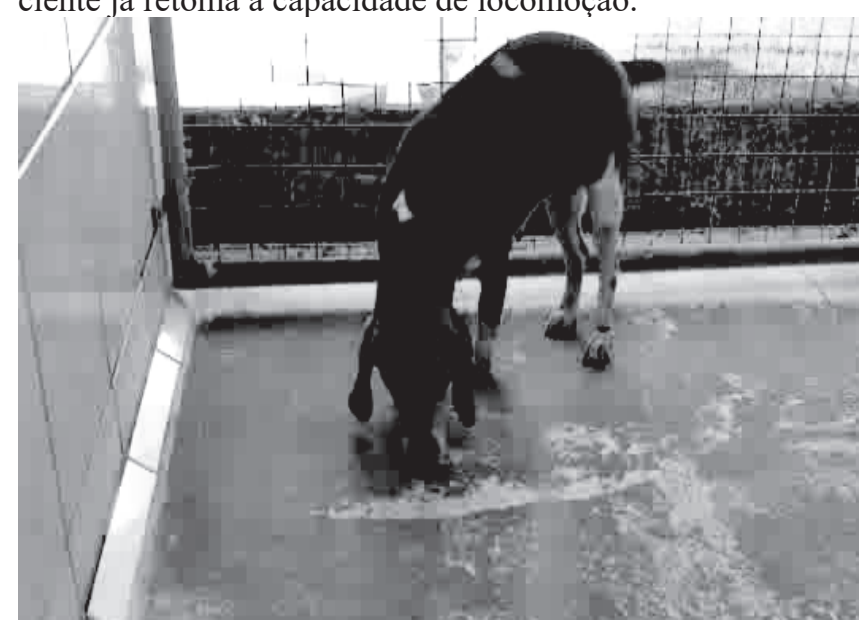

Fonte: Arquivo pessoal.

\section{Discussão}

A doença do disco intervertebral é considerada uma osteopatia ligada diretamente à genética, com raças condrodistróficas sendo as mais predispostas, e geralmente surge entre cinco e seis anos de idade (JANSSENS, 2006). Conforme a localização da doença e as suas manifestações clínicas, Wang (2009) relaciona os sintomas de discopatia toracolombar com possíveis distúrbios dos meridianos VG, VB, B e R. $\mathrm{Na}$ visão da MTC, pela teoria dos cinco elementos, o histórico e sintomas apresentados no caso relatado como a ocorrência de discopatia e consequentes déficits neurológicos em cadela de raça condrodistrófica, com seis anos de idade, que atuava como matriz reprodutora, com secreção auricular pronunciada e vocalização em gemidos são indícios de um desequilíbrio do elemento Água (SCHWARTZ, 2008). Como consequência desse desequilíbrio, a deficiência de Qi do BP se estabelece, ficando comprometida a função desse meridiano em controlar a Umidade, o que se manifesta clinicamente com diarreia e acúmulo de secreção nos ouvidos (XIE; PREAST, 2012). Por isso, os principais pontos utilizados no tratamento foram nos meridianos do R e B, os quais são regidos pela Água. Considerando os princípios Yin e Yang, o estímulo adicional com moxa foi incorporado ao tratamento devido o diagnóstico de Frio por deficiência, com o predomínio de energia Yin e deficiência de Yang. Neste relato, o Frio é clinicamente caracterizado pela ausência de exacerbação de dor ao toque (CHONGHUO; CORRAL, 1985), a coloração pálido-roxa da língua, as fezes amolecidas (XIE; PREAST, 2012) e pelo sintoma principal de incapacidade motora (LIMEHOUSE; TAYLOR-LIMEHOUSE, 2006), o qual decorre da estase de sangue e Qi gerado pelo Frio (CHONGHUO; CORRAL, 1985).

A moxabustão consiste no aquecimento dos acupontos por meio da queima da erva Artemísia vulgaris, podendo este estímulo ser de forma direta, com cones de moxa diretamente sobre a pele, ou indireta, com bastão de moxa e sem contato com a pele (ALTMAN, 2006). A escolha da terapia conjunta com moxabustão foi essencial para o sucesso do tratamento no caso apresentado, devido suas propriedades tonificante (XU; DENG; SHEN, 2014) e promotora da circulação de Qi e sangue (HUANG; SHEU, 2013). Aliás, a moxabustão é justamente indicada para quadros de deficiência (CHIU, 2013), tonificando a energia Yang e removendo o Frio e Umidade (DENG; SHEN, 2013). Conforme Chiu (2013) seriam responsáveis pelos efeitos terapêuticos da moxabustão a ação da temperatura e de fatores não térmicos como a fumaça, radiação infravermelha e propriedades farmacológicas da planta. Inclusive, diversos compostos com ampla atividade biológica têm sido identificados nas folhas e na fumaça da moxa, os quais teriam participação importante na eficácia da moxabustão. Os efeitos térmicos decorrem da ativação de receptores para calor e/ou receptores polimodais (DENG; SHEN, 2013). Alguns autores afirmam que os diferentes graus de temperatura que a moxa eleva na superfície da pele é o que determinará qual receptor será ativado (XINMIN et al., 2012 citado por WANG et al., 2013), sendo que a temperatura ótima para a moxabustão ser tolerável e apresentar a resposta terapêutica desejada deve ser num limiar maior que $43^{\circ} \mathrm{C}$ (WANG et al., 2013). A elevação da temperatura também desencadeia a degranulação de mastócitos e a queda na viscosidade e densidade sanguínea, aumentando o fluxo sanguíneo local (HUANG; SHEU, 2013).

Todavia, os mecanismos de ação que promovem os benefícios da acupuntura nas discopatias ainda não estão completamente esclarecidos. Os efeitos sobre a rigidez muscular e dor referida seriam devido à destruição de pontos-gatilho, já a analgesia pode ser consequente à liberação de endorfinas. A estimulação dos acupontos também reduziria a inflamação no local da lesão espinhal e ativaria o crescimento de axônios destruídos pela protusão/extrusão do disco intervertebral (JANSSENS, 2006). Segundo Schoen (2011), a acupuntura pode ser benéfica no tratamento de condições neurológicas em animais tanto como tratamento primário, quanto adjunto, com o prognóstico dependendo do diagnóstico, da localização e severidade das lesões presentes.

O sucesso do uso da acupuntura como tratamento eletivo do caso clínico relatado condiz com os resultados 
encontrados por diversos autores. No estudo de Hayashi (2006), observou-se uma melhora do estado neurológico, o retorno precoce da locomoção, do controle da micção e propriocepção em animais que tiveram a acupuntura adicionada ao tratamento alopático usual. Joaquim (2008) também obteve dados promissores ao avaliar 40 casos de cães com doença do disco intervertebral divididos em três grupos de tratamentos: um grupo cirurgia, um grupo eletroacupuntura e um grupo eletroacupuntura + cirurgia, com efetividade terapêutica nos índices de $40 \%, 79 \%$ e $72,7 \%$, respectivamente. A equivalência da eficácia da acupuntura e do procedimento cirúrgico no tratamento da discopatia foi relatado por Braga (2011), que ainda destacou como vantagens da acupuntura a produção de efeitos analgésico e anti-inflamatório e de ser menos onerosa e invasiva que o tratamento cirúrgico.

$\mathrm{Na}$ medicina humana, não só a acupuntura tem relatos mais frequentes na terapêutica de discopatia intervertebral, como também a moxabustão. Um exemplo disso é o estudo de Chen et al. (2012), que observaram que o uso de moxabustão no tratamento de hérnia de disco lombar em humanos foi mais efetiva que a terapêutica instituída com o anti-inflamatório diclofenaco sódico. Entretanto, os relatos do uso de moxabustão no tratamento de discopatia em cães são escassos na literatura. Janssens (2006) lista como métodos de estimulação para o tratamento desta enfermidade a puntura simples, a eletroacupuntura, a terapia a laser e a aquapuntura, porém, não faz referência a aplicação da moxa. Concomitante a essa diversidade de técnicas complementares disponíveis, outro provável motivo para a reserva ao uso da moxa pelos veterinários seria a aversão à quantidade e ao cheiro da fumaça produzida pela moxabustão. Do mesmo modo, o risco da ocorrência de queimaduras, em razão da ausência de reação do paciente com paralisia, contribuiria com a reduzida adoção da moxa no tratamento desses casos (ALTMAN, 2006). Contudo, considerando as situações específicas em que a moxa é indicada (como no caso clínico relatado - síndrome de Frio por deficiência de Yang), a praticidade e baixo custo da técnica e a resposta rápida e efetiva ao tratamento, não se justifica a limitada repercussão da aplicação de moxabustão associada à acupuntura na prática clínica veterinária. Ademais, comprovou-se que a associação da acupuntura e moxabustão como tratamento de eleição de discopatia toracolombar em cão foi efetiva no restabelecimento da saúde do paciente.

\section{Conclusão}

Os relatos da ação da acupuntura em casos de doença do disco intervertebral são instigantes, ainda mais quando se considera o fato dos tratamentos usuais implicarem dificuldades como os efeitos colaterais dos medicamentos alopáticos, os custos de uma cirurgia e os cuidados nos procedimentos pós-operatórios. Quando aplicável (dependendo do quadro clínico), a associação de acupuntura e moxabustão, técnicas terapêuticas primordiais da MTC, mostram-se como uma opção tão efetiva como as demais técnicas de estimulação desenvolvidas nos últimos anos. Considerando ainda a visão holística da MTC, os benefícios da acupuntura e moxabustão incluem não só a solução da doença manifestada, como também o re-equilíbrio do indivíduo como um todo, ajudando no restabelecimento completo da saúde e bem-estar do paciente.

\section{Referências}

ALTMAN, S. Técnicas e instrumentação. In: SCHOEN, A. M. Acupuntura veterinária: da arte antiga à medicina moderna. 2. ed. São Paulo: Roca, 2006. p. 91-108.

\section{BRAGA, V. Acupuntura no tratamento de discopatias} intervertebrais em cães. 2011. 19 f. Trabalho de Conclusão de Curso (Graduação em Medicina Veterinária) - Faculdade de Medicina Veterinária e Zootecnia da Universidade Estadual Paulista, Botucatu, 2011.

BRAUND, K. G. Degenerative and compressive structural disorders. In:_ Braund's clinical neurology in small animals: localization, diagnosis and treatment. Disponível em: <http://www.ivis.org/home.asp > Acesso em: 07 nov. 2010.

CHEN, R. et al. Heat-sensitive moxibustion for lumbar disc herniation: a meta-analysis of randomized controlled trials. Journal of Traditional Chinese Medicine, v. 32, n. 3, p. $1-2,2012$

CHIU, J. H. How does moxibustion possibly work? Evidence-Based Complementary and Alternative Medicine, v. 2013, p. 1-8, 2013.

CHONGHUO, T.; CORRAL, J. L. P. Tratado de acupuntura. Beijing: Alhambra, 1985. p. 231.

DENG, H.; SHEN, X. The Mechanism of moxibustion: ancient theory and modern research. Evidence-Based Complementary and Alternative Medicine, v. 2013, p. 1-7, 2013.

HAYASHI, A. M. Estudo clínico da eficácia da acupuntura no tratamento da discopatia tóracolombar em cães. 2006. 105 f. Dissertação (Mestrado em Clínica Cirúrgica Veterinária) - Departamento de Cirurgia, Faculdade de Medicina Veterinária e Zootecnia, Universidade de São Paulo, São Paulo, 2006.

HUANG, C.; SHEU, T. W. H. Study of the effect of moxibustion on the blood flow. International Journal of Heat and Mass Transfer, v. 63, p. 141-149, 2013.

JANSSENS, L. A. A. Acupuntura para tratar doenças de discos toracolombar e cervical. In: SCHOEN, A. M. Acupuntura veterinária: da arte antiga à medicina moderna. 2. ed. São Paulo: Roca, 2006. p. 190-195.

JOAQUIM, J. G. F. Comparação entre eletroacupuntura, cirurgia e cirurgia associada à eletroacupuntura no tratamento da doença do disco intervertebral em cães. $100 \mathrm{f}$. Tese de Doutorado (Doutorado em Medicina Veterinária) - Faculdade de Medicina Veterinária e Zootecnia da Universidade Estadual Paulista, Botucatu, 2008. 
LIMEHOUSE, J. B.; TAYLOR-LIMEHOUSE, P. A. Conceitos orientais da acupuntura. In: SCHOEN, A. $\mathrm{M}$. Acupuntura veterinária: da arte antiga à medicina moderna. 2. ed. São Paulo: Roca, 2006. p. 76-90.

MASIAN, D. S. What is new in the management of intervertebral disk disease? In: Southern European Veterinary Conference \& Congreso Nacional AVEPA, 2011, Barcelona. Proceedings... Barcelona: SEVC, 2011.

SCHOEN, A. M. Acupuncture for veterinary neurologic conditions. In: WORLD SMALL ANIMAL VETERINARY CONGRESS WSAVA, 36., Jeju. Proceedings... Jeju: WSAVA, 2011.

SCHOEN, A. M. Acupuntura veterinária: da arte antiga à medicina moderna. 2. ed. São Paulo: Roca, 2006. p. 603.

SCHWARTZ, C. Quatro patas cinco direções: um guia de medicina chinesa para cães e gatos. São Paulo: Ícone, 2008. p. 470 .

WANG, G. Y. et al. Effects of moxibustion temperature on blood cholesterol level in a mice model of acute hyperlipidemia: role of TRPV1. Evidence-Based Complementary and Alternative Medicine, v. 2013, p. 1-7, 2013.

WANG, L. P. Meridian differentiation of lumbar intervertebral disc herniation. Zhongguo Gu Shang, v. 22, n. 10, p. 777-778, 2009.

XIE, H.; PREAST, V. Medicina veterinária tradicional chinesa - princípios básicos. MedVet, São Paulo, 2012. p. 640 .

XU, J.; DENG, H.; SHEN, X. Safety of moxibustion: a systematic review of case reports. Evidence-Based Complementary and Alternative Medicine, v. 2014, p. 1-10, 2014.

Recebido em: 28.09.2015

Aceito em: 28.12.2015 